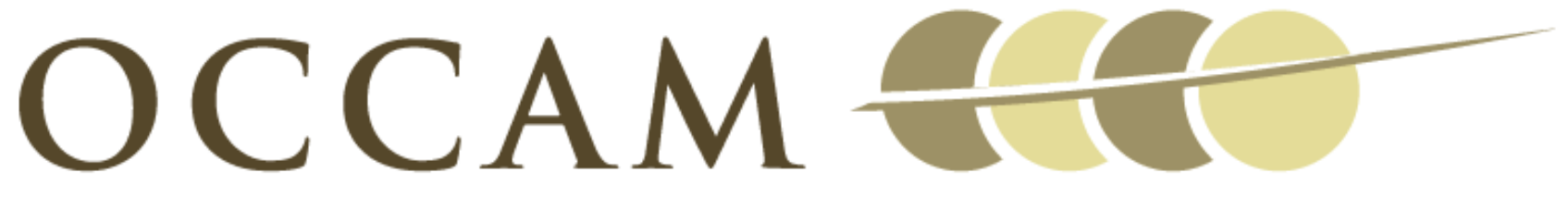

OXFORD CENTRE FOR COLLABORATIVE APPLIED MATHEMATICS

Report Number 11/70

The Capillary Interaction Between Two Vertical Cylinders by

Himantha Cooray, Pietro Cicuta and Dominic Vella

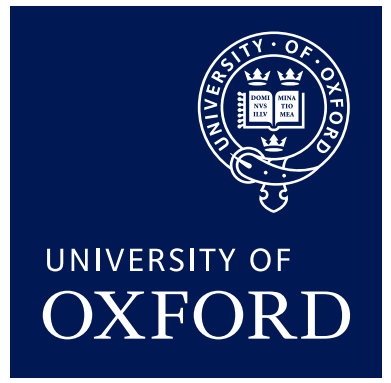

Oxford Centre for Collaborative Applied Mathematics Mathematical Institute

24 - 29 St Giles'

Oxford

OX1 3LB

England 



\title{
The Capillary Interaction Between Two Vertical Cylinders
}

\author{
Himantha Cooray ${ }^{1}$, Pietro Cicuta ${ }^{1}$ and Dominic Vella ${ }^{2}$ \\ ${ }^{1}$ Cavendish Laboratory, University of Cambridge, J J Thomson Avenue, Cambridge \\ CB3 0HE, U.K. \\ 2 Oxford Centre for Collaborative Applied Mathematics, Mathematical Institute, \\ University of Oxford, 24-29 St Giles', Oxford OX1 3LB, U.K.
}

\begin{abstract}
Particles floating at the surface of a liquid generally deform the liquid surface. Minimizing the energetic cost of these deformations results in an interparticle force, which is usually attractive and causes floating particles to aggregate and form surface clusters. Here we present a numerical method for determining the three-dimensional meniscus around a pair of vertical circular cylinders. This involves the numerical solution of the fully nonlinear Laplace-Young equation using a mesh-free finite difference method. Inter-particle force-separation curves for pairs of vertical cylinders are then calculated for different radii and contact angles. These results are compared with previously published asymptotic and experimental results. Good agreement is found between all three approaches (numerical, asymptotic and experimental) for large inter-particle separations and conditions such that the meniscus slope remains small everywhere. This is as expected since the asymptotic results were derived using the linearized Laplace-Young equation. For steeper menisci and smaller inter-particle separations, however, the numerical simulation resolves discrepancies between existing asymptotic and experimental results, demonstrating that this discrepancy was due to the nonlinearity of the Laplace-Young equation in these situations.
\end{abstract}




\section{Introduction}

An object at an otherwise planar liquid-fluid interface can distort the interface either by imposing height variations along a three-phase contact line or by imposing a nonzero derivative condition on the interface along this contact line. The first scenario is observed when the contact line is pinned to an object whilst the second scenario is observed when the contact line must deform to satisfy a contact angle condition. As a result of these deformations, two or more objects at an interface are subject to surface-mediated "capillary interactions" [1]. For floating particles, a large component of the interfacial deformation may be caused by the requirement that the vertical force components (including the particle's weight) must balance. The lateral force that results from this deformation is therefore often referred to as a "flotation force" in the literature [2] and is the mechanism behind the "Cheerios effect" [3]. Non-floating particles do not need to satisfy a vertical force balance condition; the lateral capillary force that results from interfacial deformation is usually referred to as an "immersion force" [2]. Flotation forces are observed and exploited in a range of biological systems from "meniscusclimbing" in Mesovelia [4] to pattern formation in floating mosquito egg rafts [5] while immersion forces explain some features of the collective motion of nematode worms [6]. Mankind too is learning to exploit both flotation and immersion forces, particularly as a way of self-assembling complex structures [7, 8].

The strength of the capillary interaction between two interfacial objects depends on the shape, size and surface properties of the objects in question as well as, in the case of flotation forces, their weight. Analytical and numerical solutions are straightforward only in some limiting cases, which we discuss in detail below. In this article we study the immersion forces between a pair of vertical cylinders - in some sense this is the simplest possible setup. However, we relax two of the simplifying assumptions usually made in asymptotic studies of this system; we do not require the surface gradients to remain small and we allow the cylinders to have a centre-centre separation that is comparable to their diameter. Calculating the interaction force in this simplified geometry is a first step towards finding the interaction force in more complex systems, but is also of interest in its own right: the capillary force between vertical cylinders has been measured experimentally using a torsion microbalance $[9,10]$ and, whilst generally agreeing with existing asymptotic expressions for the force [11], revealed some significant discrepancies. In this article we demonstrate that these discrepancies can largely be attributed to the simplifications made to facilitate analytical progress.

Theoretical expressions have been derived for capillary interactions between fixed vertical cylinders [11], between floating spheres [12] and between capillary multipoles [13]. However, these expressions have limited validity since they are based on the solution of the linearized Laplace-Young equation for the shape of the interface between the objects and in some cases [12] also use linear superposition of interface around a single object. These two approximations can be relaxed by solving the fully nonlinear Laplace-Young equation numerically. Previously, the finite difference method has been 


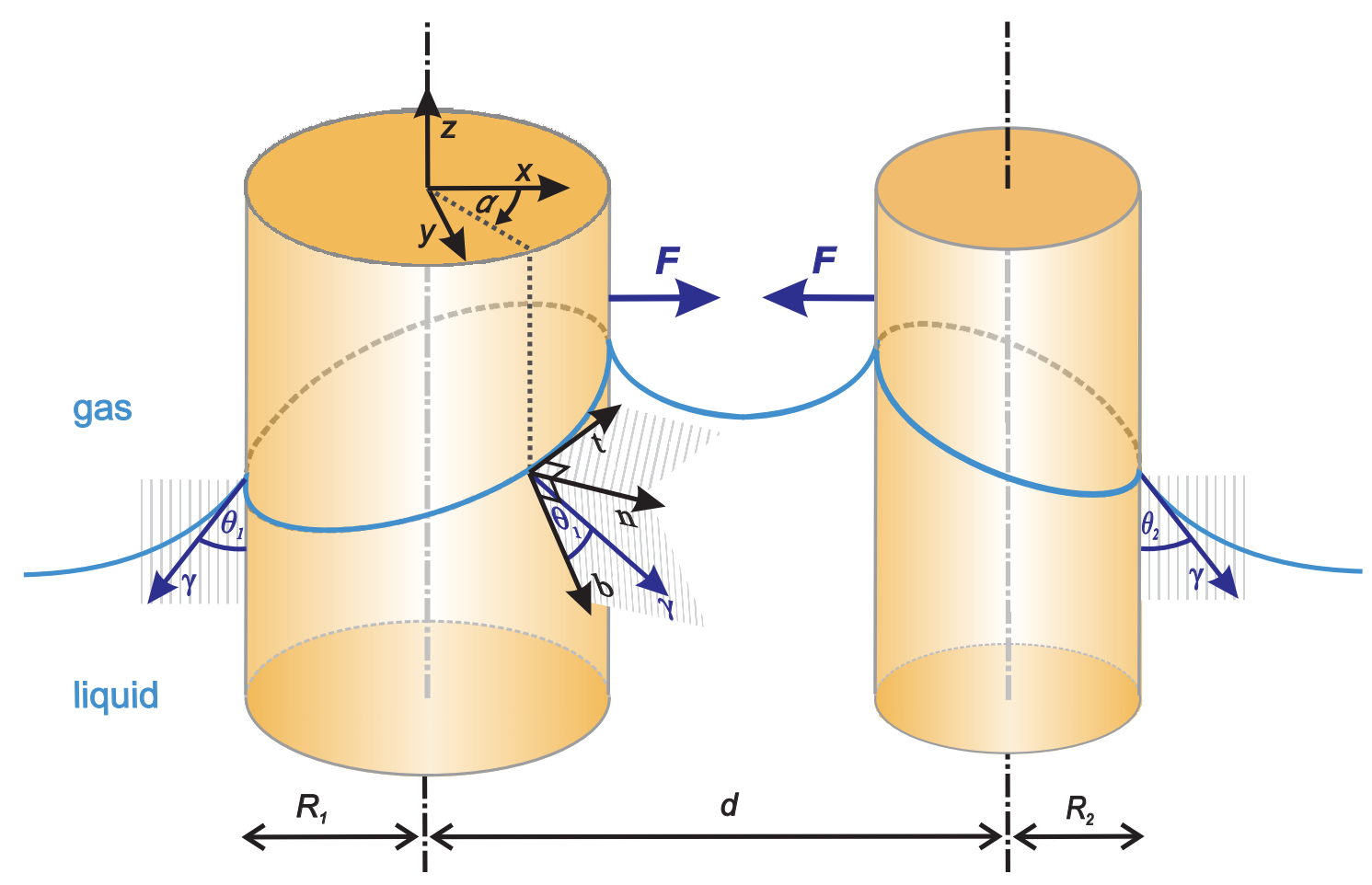

Figure 1. A pair of vertical cylinders at a liquid-fluid interface interact due to capillary forces. Boundary conditions around the two cylinders are shown.

used to provide these numerical solutions [14]. However, it was reported that numerical solution obtained in this manner was very slow and the most interesting cases of complete wetting and small inter-particle separations (for which the meniscus slope is expected to be largest and hence the validity of the asymptotic results most limited) were not studied. Indeed, for the range of parameters considered in [14] no significant deviation between the numerical solution and the asymptotic results was observed. In this paper, we discuss an implementation of the $h p$-meshless cloud method [15], which is a meshfree finite difference method. This method is chosen here for its versatility and allows the iterative solution of the nonlinear Laplace-Young equation even for conditions of complete wetting and for objects at very close range.

\section{Theoretical Setting}

We consider two vertical cylinders with radii $R_{1}, R_{2}$ and contact angles $\theta_{1}, \theta_{2}$ that pierce an otherwise planar liquid-fluid interface. This scenario is shown in figure 1 . The planar interface is disturbed by the presence of the two cylinders because of the requirement that the interface obey a contact angle condition at the surface of the cylinders. The height of the liquid interface is given by:

$$
z=\zeta(x, y)
$$


with $z=0$ representing the undisturbed liquid level. The shape of the meniscus, $\zeta(x, y)$, is described by the Laplace-Young equation, which relates $\zeta(x, y)$ to the mean curvature $\kappa$ by balancing the hydrostatic pressure in the liquid with the pressure jump due to the interfacial curvature [16]. Mathematically we may write:

$$
\zeta=\ell_{c}^{2} \kappa
$$

Here the capillary length $\ell_{c}$ is defined by:

$$
\ell_{c}=\sqrt{\frac{\gamma}{\Delta \rho g}}
$$

where $\gamma$ is surface tension and $\Delta \rho$ is density difference between the two fluids. In Cartesian coordinates, the interfacial curvature $\kappa$ is given by [14]:

$$
\kappa=\frac{\zeta_{x x}\left(1+\zeta_{x}^{2}\right)+\zeta_{y y}\left(1+\zeta_{y}^{2}\right)-2 \zeta_{x} \zeta_{y} \zeta_{x y}}{\left(1+\zeta_{x}^{2}+\zeta_{y}^{2}\right)^{\frac{3}{2}}}
$$

For a meniscus near a vertical cylinder, the boundary condition is a fixed contact angle $\theta$. As illustrated in Fig. 1, this boundary condition is simple to describe verbally but is non-trivial to express mathematically. To achieve this we define $\boldsymbol{t}$ as the running tangent to the contact line, $\boldsymbol{n}$ as the running outward normal to the cylinder wall and $\boldsymbol{b}$ as the binormal to $\boldsymbol{t}$ and $\boldsymbol{n}$. Then the contact angle is set with respect to the plane containing $\boldsymbol{n}$ and $\boldsymbol{b}$ [1]. If $\hat{\gamma}$ is the unit tangent to the liquid interface on this plane and $\hat{\boldsymbol{b}}$ is the unit binormal, then the contact angle boundary condition reads:

$$
\hat{\boldsymbol{b}} \cdot \hat{\gamma}=\cos \theta_{i}
$$

on the surface of cylinder $i$.

The solution of the Laplace-Young equation (2) subject to the boundary condition (5) on the surface of each cylinder, and the requirement that $\zeta(x, y) \rightarrow 0$ as $x, y \rightarrow \pm \infty$, is sufficient to determine the meniscus profile $\zeta(x, y)$ for a particular cylinder separation $d$ and given cylinder radii $R_{1,2}$ and contact angles $\theta_{1,2}$.

The quantity of most interest in this paper is the lateral interaction force $F$ between the cylinders i.e. the force that must be applied to each cylinder to maintain a particular separation $d$. In general, we expect that the two cylinders will be attracted to one another (so that $F>0$ ) but we shall see that there are circumstances under which a repulsive force exists at large separations. Once the shape of the meniscus is known, the component of the force of attraction due to surface tension can be calculated by integrating the force due to surface tension around the contact line, as suggested in [11]. The surface tension force acting on a cylinder is given by:

$$
\boldsymbol{F}_{\gamma}=\oint \gamma \mathrm{d} s
$$

Here $\boldsymbol{\gamma}$ is the surface tension force acting along an infinitesimal arclength $\mathrm{d} s$ along the contact line, and $\mathrm{d} s$ is given by:

$$
\mathrm{d} s=\chi \mathrm{d} \alpha
$$


where $\chi$ is defined by

$$
\chi=\sqrt{R_{k}^{2}+\left(\frac{\partial \zeta}{\partial \alpha}\right)^{2}}, \quad k=1,2,
$$

and $\alpha$ is the angle between the $x$-axis and the line joining the centre of the cylinder to a point on the contact line. (See Fig. 1.)

$\gamma$ can be decomposed as as

$$
\boldsymbol{\gamma}^{(i)}=\gamma\left(\hat{\boldsymbol{b}} \cos \theta_{i}+\hat{\boldsymbol{n}} \sin \theta_{i}\right), i=1,2 .
$$

If $\boldsymbol{e}_{\boldsymbol{x}}, \boldsymbol{e}_{\boldsymbol{y}}$ and $\boldsymbol{e}_{\boldsymbol{z}}$ are unit vectors along the $x, y$ and $z$ directions, respectively, then:

$$
\begin{aligned}
\hat{\boldsymbol{n}} & =\cos \alpha \boldsymbol{e}_{\boldsymbol{x}}+\sin \alpha \boldsymbol{e}_{\boldsymbol{y}} ; \\
\hat{\boldsymbol{t}} & =\frac{1}{\chi}\left(R_{k} \sin \alpha \boldsymbol{e}_{\boldsymbol{x}}-R_{k} \cos \alpha \boldsymbol{e}_{\boldsymbol{y}}-\frac{\partial \zeta}{\partial \alpha} \boldsymbol{e}_{\boldsymbol{z}}\right) ; \\
\hat{\boldsymbol{b}} & =\hat{\boldsymbol{n}} \times \hat{\boldsymbol{t}} \\
& =\frac{1}{\chi}\left(-\frac{\partial \zeta}{\partial \alpha} \sin \alpha \boldsymbol{e}_{\boldsymbol{x}}+\frac{\partial \zeta}{\partial \alpha} \cos \alpha \boldsymbol{e}_{\boldsymbol{y}}-R_{k} \boldsymbol{e}_{\boldsymbol{z}}\right) .
\end{aligned}
$$

Substituting into Eq. (9), the $x$ component of $\boldsymbol{\gamma}$ becomes:

$$
\gamma_{x}^{(i)}=\gamma\left(-\frac{\partial \zeta}{\partial \alpha} \frac{1}{\chi} \sin \alpha \cos \theta_{i}+\cos \alpha \sin \theta_{i}\right)
$$

Combination of Eq. (13) with Eq. (6) and Eq. (7) gives:

$$
F_{\gamma, x}^{(i)}=\gamma \int_{0}^{2 \pi}\left(-\frac{\partial \zeta}{\partial \alpha} \sin \alpha \cos \theta_{i}+\chi \cos \alpha \sin \theta_{i}\right) \mathrm{d} \alpha .
$$

In addition to the force from surface tension, a force also acts on each cylinder due to the variations in hydrostatic pressure around the cylinder. This force arises from the differences in liquid level around the cylinder. The $x$-component of this force is given by:

$$
F_{p, x}=\Delta \rho g R_{k} \int_{0}^{2 \pi} \frac{1}{2} \zeta^{2} \cos \alpha \mathrm{d} \alpha .
$$

Then the total force of attraction between the two cylinders is:

$$
F_{x}=F_{\gamma, x}^{(i)}+F_{p, x}^{(i)} .
$$

It should be noted that $F_{\gamma, x}^{(1)}$ and $F_{\gamma, x}^{(2)}$ are not necessarily equal. However, the total force given by Eq. (16) is the same for both cylinders according to Newton's third law.

The above approach is used to calculate the force of interaction from our numerical solution from the meniscus shape. The force can also be calculated using an energy approach, as used in the asymptotic solutions discussed in $§ 3$. This approach is discussed in detail in [11]. If the total energy of the system is $E$ and the inter-particle centre-tocentre distance is $d$, then the inter-particle attractive force is given by:

$$
F=\frac{\mathrm{d} E}{\mathrm{~d} d}
$$


The interaction energy $E$ is comprised of three components as shown below:

$$
\begin{aligned}
E & =\Delta \rho g \int_{S_{p}} \frac{1}{2} \zeta^{2} \mathrm{~d} S \\
& +\sum_{k=1}^{2}\left(\gamma_{s l}^{(k)}-\gamma_{s v}^{(k)}\right) \int_{0}^{2 \pi} \zeta(\alpha, k) R_{k} \mathrm{~d} \alpha \\
& +\gamma \int_{S_{p}} \sqrt{1+\zeta_{x}^{2}+\zeta_{y}^{2}} \mathrm{~d} S .
\end{aligned}
$$

In Eq. (18), the first term represents the gravitational potential energy of the fluids, the second term is the interfacial energy between the walls of the cylinders and each of the two fluids, and the third term is the interfacial energy between the two fluids. Here $S_{p}$ is the projected area of the fluids to the horizontal plane, $\gamma_{s l}^{(k)}$ is the is the surface tension between the cylinder $k$ surface and the lower liquid, $\gamma_{s v}^{(k)}$ is the is the surface tension between the cylinder $k$ surface and the upper fluid, $\zeta(\alpha, k)$ is the height of the contact line around cylinder $k$ where $\alpha$ is the angle measured around a cylinder.

\section{Existing Asymptotic Results}

\subsection{Some simplifications}

There are two asymptotic limits in which the interaction force between two cylinders can be solved analytically, as described below. In both of these limits, the problem is simplified by linearisation of the Laplace-Young equation so that the interfacial deformation is approximately given by:

$$
\zeta=\ell_{c}^{2} \nabla^{2} \zeta
$$

This approximation is valid when the meniscus slopes are small:

$$
\frac{\partial \zeta}{\partial x} \ll 1, \frac{\partial \zeta}{\partial y} \ll 1
$$

When calculating the energy of interaction in certain asymptotic limits, it is furthermore expedient to neglect the gravitational energy of the liquid in Eq. (18) compared to other energies. This approximation is expected to be valid for small cylinders $R_{1}, R_{2} \ll \ell_{c}$. We note that although we neglect the gravitational energy in the asymptotic results that follow, our numerical simulations (discussed in $§ 4$ ) do not neglect this energy.

However, it is also important to emphasize that the inclusion of the term linear in $\zeta$ in (19) is important in the asymptotic results that follow since it is this term that ensures that the interface decays to the undisturbed level far from the cylinders something that the solution to Laplace's equation $\nabla^{2} \zeta=0$ is unable to do.

\subsection{Long-Range Asymptotic Solution}

If the separation of the two cylinders $d$ is large compared to the capillary length $\ell_{c}$, we may consider the meniscus around a single cylinder and, assuming that the individual 
menisci may simply be superposed, calculate the surface energy increase that results from their interaction. This is the dominant energy that leads to the force of interaction.

A detailed solution of the meniscus shape around a single cylinder was presented by Lo [17]. Far away from the cylinder (compared to the capillary length $\ell_{c}$ ) it is convenient to write the linearized Laplace-Young equation (19) in cylindrical polar coordinates to give the interface shape $\zeta(r)$ as the solution of:

$$
\zeta=\frac{\ell_{c}^{2}}{r} \frac{\partial}{\partial r}\left(r \frac{\partial \zeta}{\partial r}\right)
$$

where $r$ is radial distance measured from the centre of the cylinder. The solution of this equation that decays as $r \rightarrow \infty$ is:

$$
\zeta(r)=A K_{0}\left(r / \ell_{c}\right)
$$

where $K_{0}$ is the modified Bessel function of the first kind of zeroth order [18]. In the limit $R \ll 1$ we may determine the constant of integration $A$ as follows. The weight of liquid lifted up within the meniscus is

$$
W=2 \pi \rho g \int_{R}^{\infty} r \zeta \mathrm{d} r=2 \pi \gamma A \frac{R}{\ell_{c}} K_{1}\left(R / \ell_{c}\right) \sim-2 \pi \gamma A \quad\left(R \ll \ell_{c}\right) .
$$

Using the generalized Archimedes' principle [19] this weight must be supported by the force of surface tension acting around the contact line, which in this case is $2 \pi R \times \gamma \cos \theta$. Hence we find that $A=R \cos \theta$. We note that this result may also be obtained by careful matching of the profile from Eq. (22) with the meniscus shape near to the cylinder for which the slope cannot necessarily be assumed to be small [17].

Assuming that the two cylinders are sufficiently far apart from one another (i.e. that their separation $d \gg \ell_{c}$ ) then we neglect the first and last terms of Eq. (18), and expect that the energy of interaction will simply be the increase in surface energy caused by the change in the height of the meniscus on cylinder 2 due to the meniscus of cylinder 1 (and vice versa). This energy is larger than the increase in gravitational potential energy of the meniscus due to the same effect and the change in energy of the liquidfluid interface, provided that $R_{1,2} \ll \ell_{c}$. Since this energy of interaction $\Delta E$ is caused by solid-vapour interface being replaced by solid-liquid interface we expect that the difference in the surface energies of these interfaces on cylinder $2, \gamma_{s l}^{(2)}-\gamma_{s v}^{(2)}$, will be important and so we have:

$$
\Delta E=2 \pi R_{2}\left[\gamma_{s l}^{(2)}-\gamma_{s v}^{(2)}\right] \zeta_{1}(d)
$$

Using Young's law $\gamma_{s l}^{(2)}-\gamma_{s v}^{(2)}=\gamma \cos \theta_{2}$ and Eq. (22) for the meniscus profile $\zeta_{1}$, we find that:

$$
\Delta E=2 \pi \gamma R_{1} R_{2} \cos \theta_{1} \cos \theta_{2} K_{0}\left(d / \ell_{c}\right) .
$$

The force of attraction $F$ is given by $F=\mathrm{d} E / \mathrm{d} d$ and so we have that the force of interaction between the two cylinders is:

$$
F=\frac{2 \pi \gamma R_{1} R_{2}}{\ell_{c}} \cos \theta_{1} \cos \theta_{2} K_{1}\left(d / \ell_{c}\right)
$$

This result is given by several authors (see for example [2]) and can be derived more formally. However, the result Eq. (25) remains unchanged. 


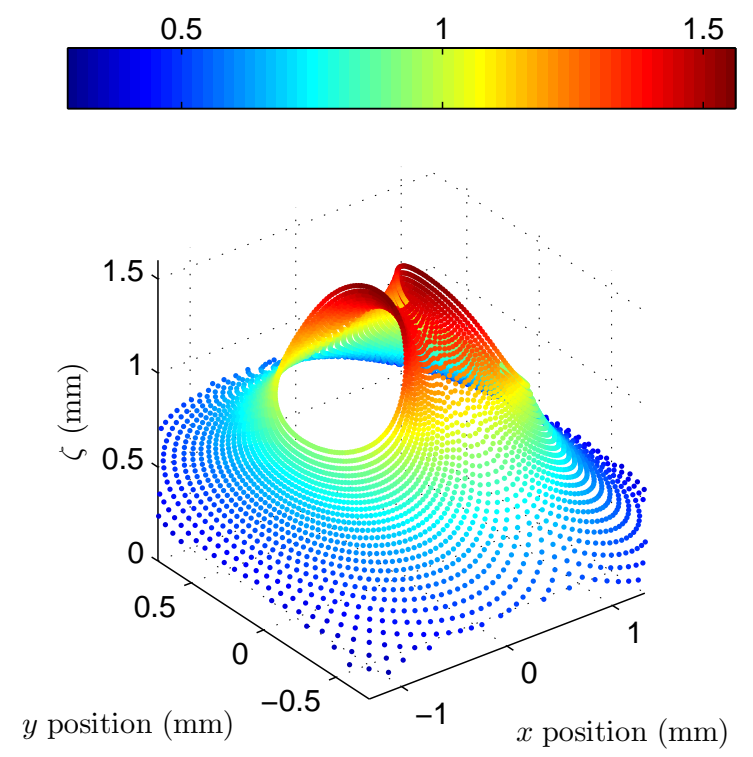

Figure 2. Shape of the 3-dimensional meniscus near two cylinders with $R_{1}=R_{2}=$ $356 \mu \mathrm{m}, d=1100 \mu \mathrm{m}$ and $\theta=0^{\circ}$ in a solution of SDS with $\gamma=36.8 \mathrm{mN} / \mathrm{m}$, $\rho=1000 \mathrm{~kg} / \mathrm{m}^{3}$ so that $\ell_{c}=1.9 \mathrm{~mm}$

\subsection{Short-Range Asymptotic Solution}

Kralchevsky et al. [11] developed another analytical solution for a pair of cylinders in the opposite limit in which the inter-particle distance is small compared to the capillary length: $d \ll \ell_{c}$. Instead of linear superposition, the linearized Laplace-Young equation is solved in bipolar coordinates $(\tau, \sigma)$ for the meniscus around two vertical cylinders. We do not repeat this involved calculation here but merely note the final result for the force between two identical cylinders:

$$
F=\frac{2 \gamma \pi R^{2} \cos ^{2} \theta}{\sqrt{d^{2}-4 R^{2}}}
$$

We note that Eq. (26) is strictly valid only in the limit $R \ll d \ll \ell_{c}$ and so:

$$
F \approx \frac{2 \gamma \pi R^{2} \cos ^{2} \theta}{d}
$$

Furthermore, we note that this expression is precisely the $d \ll \ell_{c}$ limit of the long range force law Eq. (25). To make best use of this observation, Kralchevsky \& Nagayama [2] pose the composite expansion:

$$
F=\frac{2 \pi \gamma R_{1} R_{2}}{\ell_{c}} \cos \theta_{1} \cos \theta_{2} K_{1}\left(d / \ell_{c}\right), \quad\left(R \ll \ell_{c}\right) .
$$

\section{Numerical Solution}

Using numerical methods it is possible to solve the full nonlinear Laplace-Young equation - Eq. (2) without the simplifying assumptions needed to make analytical progress. However, this requires a careful choice of numerical method. We first 
attempted to implement a standard finite difference method based on a uniform Cartesian grid. This did not yield satisfactory results for two main reasons: (a) the mis-match between the square grid and the exact circular boundary and (b) uniform grids are inefficient at simultaneously dealing with the rapid change in interface shape near the cylinder and slower change further away - it is better to have a fine mesh there, which gradually coarsens towards the outer boundary. The $h p$-Meshless Cloud Method [15] eliminates both of these issues. It does not require a regular grid, but only a collection of nodes. These nodes can easily be positioned on the domain boundaries (the cylinders) with nodes within the domain distributed on concentric circles, centred at the centre of each cylinder. (These circles are clipped along the centre-line between the two circles to avoid them intersecting one another.) Furthermore, the radii of the grid circles are selected in such a way that the node density decreases exponentially with distance from the cylinders.

The nonlinear terms in the Laplace-Young equation are handled using an iterative scheme. The meniscus height at the $(N-1)^{\text {th }}$ iteration is used to give estimates of the nonlinear terms. This allows a linear equation for the meniscus height at a particular point for the $N^{\text {th }}$ iteration to be written, namely:

$$
\zeta^{[N]}=\ell_{c}^{2} \frac{\zeta_{x x}^{[N]}\left(1+\zeta_{x}^{2}\right)^{[N-1]}+\zeta_{y y}^{[N]}\left(1+\zeta_{y}^{2}\right)^{[N-1]}-2 \zeta_{x y}^{[N]}\left(\zeta_{x} \zeta_{y}\right)^{[N-1]}}{\left[\left(1+\zeta_{x}^{2}+\zeta_{y}^{2}\right)^{\frac{3}{2}}\right]^{[N-1]}} .
$$

It is also necessary to modify Eq. (5) so that the relevant nonlinearities are handled by a similar iterative procedure. If $\hat{\boldsymbol{s}}$ is the unit normal to the liquid surface from the contact point along the plane containing $\boldsymbol{b}$ and $\boldsymbol{n}$, it can be deduced from Eq. (5) that:

$$
\hat{\boldsymbol{n}} \cdot \hat{\boldsymbol{s}}=\cos \theta_{i} .
$$

Now

$$
\{\hat{\boldsymbol{s}}\}=\left\{\zeta_{x}, \zeta_{y},-1\right\} / \sqrt{\zeta_{x}^{2}+\zeta_{y}^{2}+1}
$$

and we may write $\hat{\boldsymbol{n}}$

$$
\{\hat{\boldsymbol{n}}\}=\left\{n_{x}, n_{y}, 0\right\}
$$

since $\hat{\boldsymbol{n}}$ lies in the horizontal plane. Eq. (29) can then be expressed as:

$$
\zeta_{x} n_{x}+\zeta_{y} n_{y}=\cos \theta_{i} \sqrt{\zeta_{x}^{2}+\zeta_{y}^{2}+1}
$$

which may in turn be converted to an iterative equation

$$
\left[\zeta_{x} n_{x}+\zeta_{y} n_{y}\right]^{[N]}=\cos \theta_{i}\left[\sqrt{\zeta_{x}^{2}+\zeta_{y}^{2}+1}\right]^{[N-1]} .
$$

For each node in the domain, a "star" of nearby nodes are selected. This is done by dividing a large circle drawn from the central node into equal sections, and selecting an equal number of nodes from each section. Using the moving least squares (MLS) approximation [20], all the derivatives needed for the iterative scheme described by Eq. (28) and Eq. (33) may be determined as linear combinations of the values of $\zeta$ of 
the nodes in the star. This determination of the linear combinations is slightly complex if a star contains nodes on the cylinder boundaries. In this case, the stars are not properly balanced. To avoid this, an additional degree of freedom is given for the boundary nodes, as recommended in [15]. This is done by adding Eq. (33) into the MLS approximation. Using these linear combinations, Eq. (28) may be expressed for each node; yielding a system of linear equations for the whole domain, which can be expressed as a matrix equation. The solution of this matrix equation amounts to one iteration of Eq. (28) and Eq. (33). The addition of Eq. (33) to the MLS approximation makes sure that the boundary nodes satisfy both the boundary condition and the Laplace-Young equation.

This iterative scheme is continued until the maximum difference between the liquid levels in two consecutive iterations is very small (i.e. on the order of $10^{-13} \mathrm{~m}$ ). Starting from an initially flat interface (valid for $\theta=90^{\circ}$ ), the contact angle was gradually changed from $90^{\circ}$ to the desired contact angle. The final converged solution of Eq. (28) and Eq. (33) is the shape of the meniscus. A typical interface shape, showing the meniscus height calculated at each node point is shown in Fig. 2. With this converged meniscus shape, the force of interaction was determined by integration along the 3-phase contact lines as described by Eqs. (6-16).

If the iterative scheme failed to converge, the collection of nodes was refined by adding more nodes in the areas of maximum error, according to the $h$-adaptive method proposed in [21]. The typical distance from the centre of a cylinder to the edge of the domain was $20 \mathrm{~mm}$, and a typical domain contained 6000-9000 nodes.

\section{Results and Discussion}

Fig. 3(a) and Fig. 3(b) show the capillary attractive force between two perfectly wetting cylinders (i.e. $\theta=0^{\circ}$ ). Over the whole range of available experimental data, the numerical solution agrees very well with experiments [9]. In addition, the asymptotic solution (27) agrees with both numerics and experiments at large interparticle separations, as expected. However, when the two cylinders are close to one another, this asymptotic solution deviates from the experimental data. We attribute this discrepancy to the fact that at such small separations the meniscus slopes are significant, invalidating the linearization of the Laplace-Young equation made in the derivation of Eq. (25). The high meniscus slopes present for these parameter values is clearly illustrated in the numerically generated meniscus profile shown in Fig. 2.

It is well-known that the meniscus around a single very small cylinder $\left(R \ll \ell_{c}\right)$ decays over a distance comparable to the cylinder radius $R$, rather than the capillary length $\ell_{c}$ [17]. Because of this we expect that the asymptotic force law Eq. (27) should be valid for cylinder separations $d$ satisfying $R \ll d$. To confirm this expectation, Fig. 4 shows the results of numerical solutions in this regime. We observe that as $d \rightarrow 2 R$ the asymptotic result Eq. (27) breaks down because of the large meniscus slopes (as previously) but that for $d \gg R$ the asymptotic expression is perfectly adequate.

As a more compelling demonstration of the predictive power of our numerical 

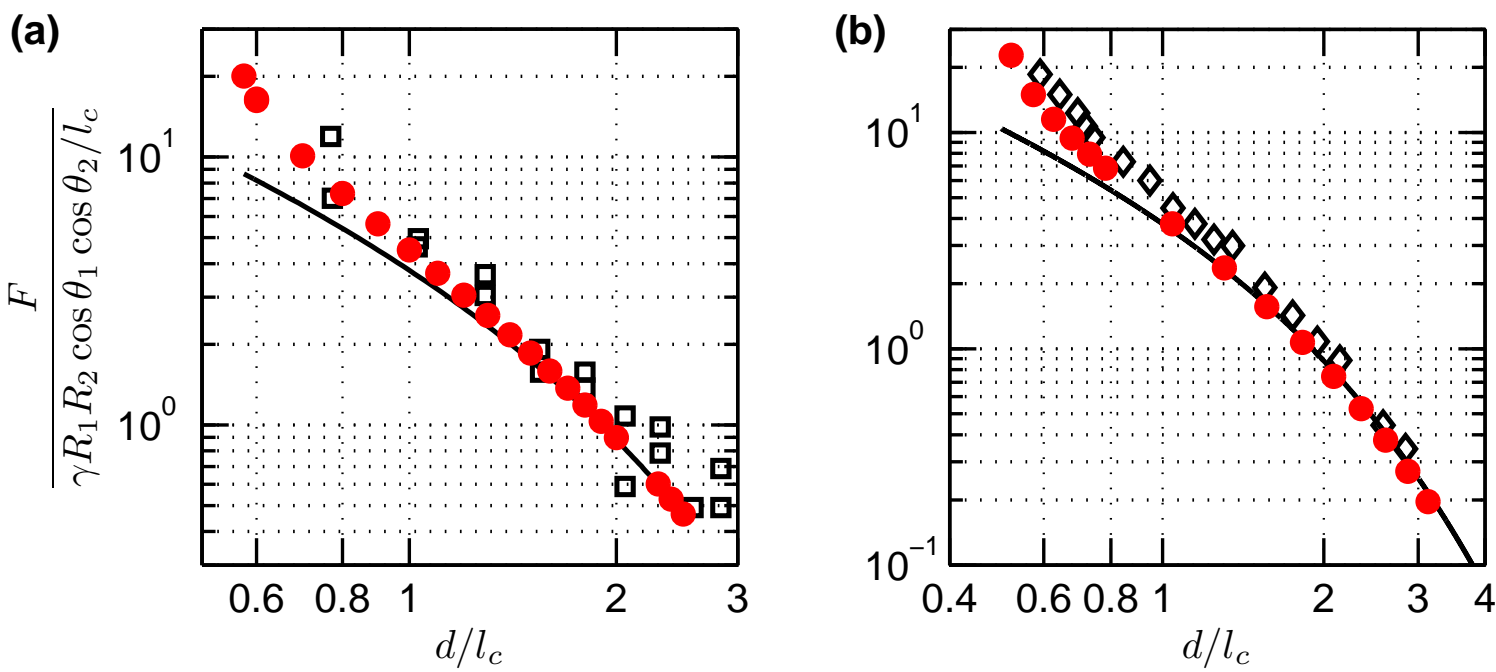

Figure 3. (a) Force of attraction between two identical hydrophilic cylinders with $\theta_{1}=\theta_{2}=0^{\circ}$ and $R_{1}=R_{2}=360 \mu \mathrm{m}$ in a solution of SDS with $\gamma=36.8 \mathrm{mN} / \mathrm{m}$, $\rho=1000 \mathrm{~kg} / \mathrm{m}^{3}$ so that $\ell_{c}=1.9 \mathrm{~mm}$. Experimental results from [9] ( $\square$ ) are shown along with predictions from the full numerical solution described here $(\bullet)$ and the asymptotic result Eq. (27) (solid curve). (b) Force of attraction between two similar, but not identical, hydrophilic cylinders with $\theta_{1}=\theta_{2}=0^{\circ}, R_{1}=315 \mu \mathrm{m}$ and $R_{2}=370 \mu \mathrm{m}$ in the same SDS solution as in (a). Experimental results from [10] $(\diamond)$ are shown along with predictions from the full numerical solution described here (•) and the asymptotic result Eq. (27) (solid curve).

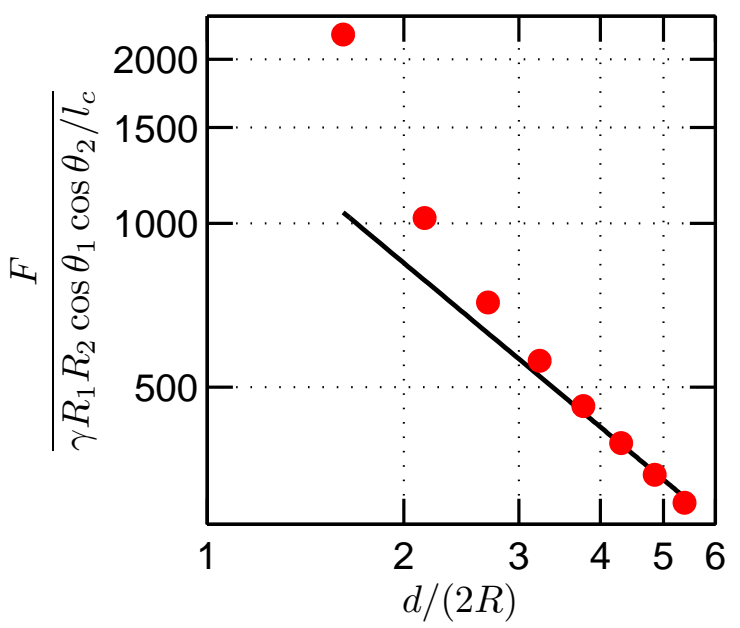

Figure 4. The asymptotic solution Eq. (27) (solid curve) agrees with numerical simulations for $R \ll d \ll \ell_{c}$ (points). Here $R_{1}=R_{2}=5 \mu \mathrm{m}, \theta_{1}=\theta_{2}=0^{\circ}$ and the liquid properties are those for an air-water interface (i.e. $\gamma=72.4 \mathrm{mN} / \mathrm{m}$ and $\rho=1000 \mathrm{~kg} / \mathrm{m}^{3}$ so that $\left.\ell_{c}=2.7 \mathrm{~mm}\right)$. The cylinder diameter here is $2 R \approx 3.7 \times 10^{-3} \ell_{c}$.

method in comparison to the asymptotic result Eq. (27), Fig. 5 shows the interaction force between a hydrophilic and a hydrophobic cylinder. In this situation, the asymptotic 


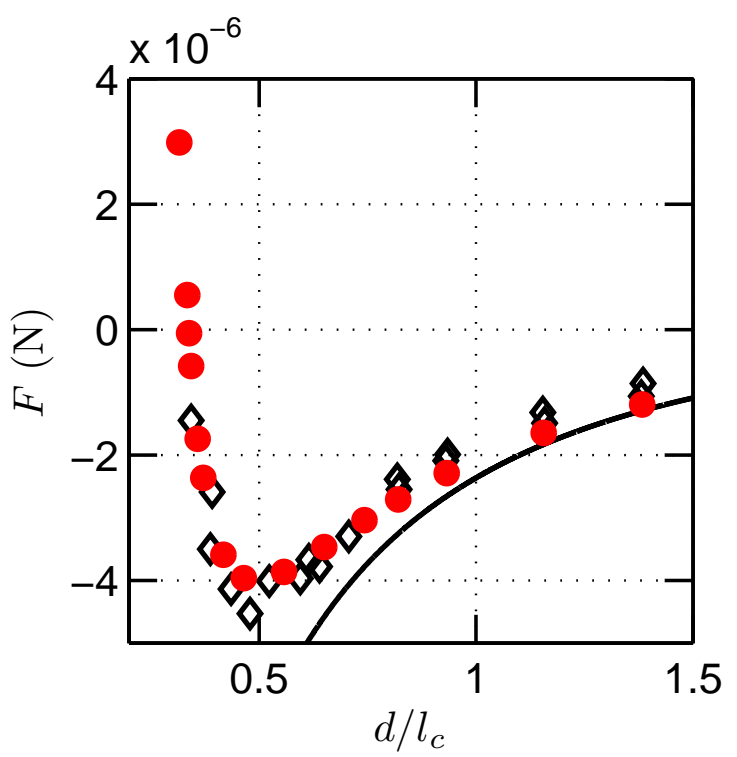

Figure 5. Force of interaction between two dissimilar cylinders: one hydrophilic and one hydrophobic in water with $\gamma=72.4 \mathrm{mN} / \mathrm{m}, R_{1}=370 \mu \mathrm{m}, R_{2}=315 \mu \mathrm{m}$ and $\theta_{1}=0^{\circ}$. (The value of $\theta_{2}$ is not reported.) Numerical results $(\bullet)$ are shown together with the asymptotic prediction Eq. (27) (solid curve) with the value $\theta_{2}=101.5^{\circ}$. The numerical simulation is able to reproduce the reduction in repulsive force observed at short ranges in the experimental results $(\diamond)[10]$ and shows that the force becomes attractive at very short range. According to [10], the long-range asymptotic result agrees best with experiments when $\theta_{2}=99^{\circ}$. However, carrying out the numerical solution for a range of $\theta$ (with increments of $0.5^{\circ}$ ), we found that the best match is $\theta_{2}=101.5^{\circ}$.

result is able to predict the long range repulsive force that is observed experimentally $(F<0)$. However, it is completely unable to reproduce the fact that this repulsion changes sign and becomes a short range attraction when the cylinder separation becomes on the order of their radii. A physical explanation of this transition to attraction was given by Poynting and Thomson for two vertical plates and is repeated in ref. [3].

Finally, we discuss the validity of approximating the true force law by a power law. Such approximations are of considerable use in more complex scenarios, most notably when attempting to understand the dynamics of aggregation [3, 12, 22]. Based on the composite asymptotic expansion Eq. (27) it is common to use the well-known result [18] that $K_{1}(x) \sim x^{-1}$ for $x \ll 1$ to obtain $F \sim d^{-1}$ for $d \ll \ell_{c}$. Fig. 6 shows the effective power-law exponent determined from the numerical simulations presented in this paper. We observe two features of this power-law exponent: firstly, the value of the power-law is not universal and depends both on $d / \ell_{c}$ and the particle size $R / \ell_{c}$. Secondly, the regime of validity of such power-laws is extremely limited with none of those plotted in Fig. 6 valid over even a decade in $d / \ell_{c}$ ! This should serve as a warning when making such simplifications or when trying to infer a simple exponent from experimental data. 


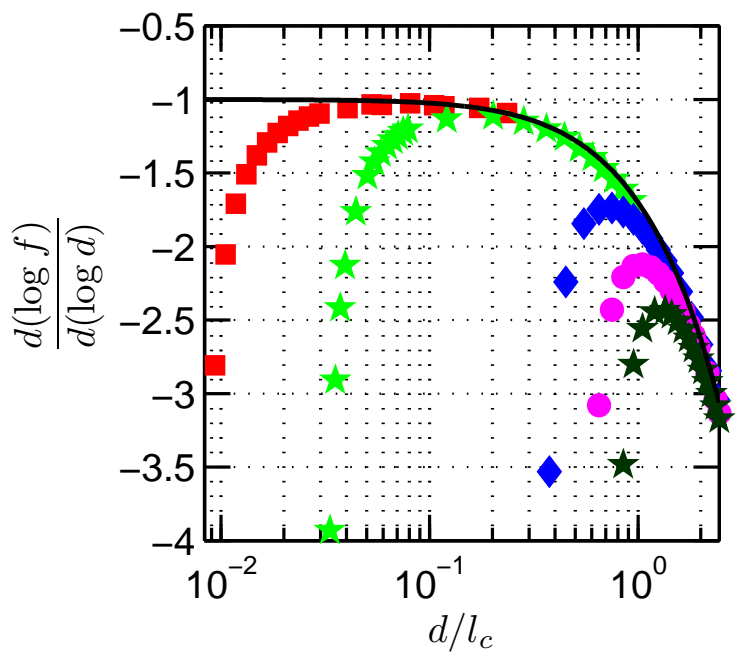

Figure 6. The force-law is not well approximated by a universal power law. The local power-law exponent is determined as the logarithmic derivative of the numericallydetermined force with respect to distance. Here we consider pairs of identical cylinders with $\theta=0^{\circ}$ and a variety of radii: $R=0.0026 \ell_{c}(\boldsymbol{\square}), R=0.01 \ell_{c}(\star), R=0.11 \ell_{c}$ $(\diamond), R=0.16 \ell_{c}(\mathbf{\bigcirc}), R=0.26 \ell_{c}(\star)$. The result expected on the basis of the asymptotic solution Eq. (27) is shown for comparison (solid curve) and demonstrates the oft-assumed exponent of -1 in the limit $d \ll \ell_{c}$.

\section{Conclusions}

We have presented numerical solutions of the nonlinear Laplace-Young equation based on the $h p$-Meshless Cloud method. This provides a versatile tool for the simulation of the meniscus shape around pairs of vertical cylinders in regimes where simple asymptotic approximations are not valid. Crucially, the determination of the interface shape via this method allowed us to calculate the force-separation curve for a range of parameter values and to probe the regime of validity of the well-known asymptotic solutions. These asymptotic solutions rely on the linearization of the Laplace-Young equation and hence fail in situations where the meniscus slope cannot be assumed to be small. In particular, this simplification is not valid for contact angles near perfect wetting and/or complete hydrophobicity, and when the two objects are close to each other. In these regions, the full numerical solution is necessary. Our numerical simulations show excellent agreement with experimental results from the literature even in regimes where the asymptotic results are at variance with experiments. Finally, we demonstrated that the true interaction force-law is not well approximated by simple power laws and hence that caution should be used when making such approximations.

\section{Acknowledgments}

We thank Dr. J. Li and Dr. M. Oettel for many useful discussions. This publication is based on work supported in part by Developing World Education Fund Cambridge 
Scholarship made by Cambridge Commonwealth Trust, Overseas Research Students Awards Scheme (H.C.), and Grant No. KUK-C1-013-04 made by King Abdullah University of Science and Technology (KAUST) (D.V.).

\section{References}

[1] B.P. Binks and T. S. Horozov. Colloidal particles at liquid interfaces. Cambridge University Press, Cambridge (U.K.), 2006.

[2] P. A. Kralchevsky and K. Nagayama. Particles at Fluid Interfaces and Membranes: Attachment of colloid particles and proteins to interfaces and formation of two-dimensional arrays. Elsevier, Amsterdam, 2001.

[3] D. Vella and L. Mahadevan. The Cheerios Effect. Am. J. Phys., 73:817-825, 2005.

[4] D. L. Hu and J. W. M. Bush. Meniscus-climbing insects. Nature, 437:733-736, 2005.

[5] Z. Saliternik. The macroscopic differentiation of anopheline eggs according to their pattern on the surface of water. Bull. Entomol. Rev., 33:221-222, 1942.

[6] S. Gart, D. Vella, and S. Jung. The collective motion of nematodes in a thin liquid layer. Soft Matter, 7:2444-2448, 2011.

[7] N. B. Bowden, A. Terfort, J. Carbeck, and G. M. Whitesides. Self-assembly of mesoscale objects into ordered two-dimensional arrays. Science, 276:233-235, 1997.

[8] B. Pokroy, S. H. Kang, L. Mahadevan, and J. Aizenberg. Self-organization of a mesoscale bristle into ordered, hierarchical helical assemblies. Science, 323:237-240, 2009.

[9] C. D. Dushkin, P. A. Kralchevsky, V. N. Paunov, H. Yoshimura, and K. Nagayama. Torsion balance for measurement of capillary immersion forces. Langmuir, 12(3):641-651, January 1996.

[10] O. D. Velev, N. D. Denkov, V. N. Paunov, P. A. Kralchevsky, and K. Nagayama. Direct measurement of lateral capillary forces. Langmuir, 9(12):3702-3709, December 1993.

[11] P. A. Kralchevsky, V. N. Paunov, N. D. Denkov, I. B. Ivanov, and K. Nagayama. Energetical and force approaches to the capillary interactions between particles attached to a liquid-fluid interface. Journal of Colloid and Interface Science, 155(2):420 - 437, 1993.

[12] D. Y. C. Chan, J. D. Henry, and L. R. White. The interaction of colloidal particles collected at fluid interfaces. Journal of Colloid and Interface Science, 79(2):410 - 418, 1981.

[13] K. D. Danov, P. A. Kralchevsky, B. N. Naydenov, and G. Brenn. Interactions between particles with an undulated contact line at a fluid interface: Capillary multipoles of arbitrary order. Journal of colloid and interface science, 287(1):121-134, 2005.

[14] C. Pozrikidis. Computation of three-dimensional hydrostatic menisci. IMA Journal of Applied Mathematics, 75:418-438, 2010.

[15] T. J. Liszka, C. A. M. Duarte, and W. W. Tworzydlo. hp-meshless cloud method. Computer Methods in Applied Mechanics and Engineering, 139(1-4):263 - 288, 1996.

[16] R. Finn. Capillary surface interfaces. Notices of the AMS, 46:770-781, 1999.

[17] L. L. Lo. The meniscus on a needle - a lesson in matching. J. Fluid Mech., 132:65-78, 1983.

[18] M. Abramowitz and I. A. Stegun. Handbook of Mathematical Functions with Formulas, Graphs, and Mathematical Tables. Dover, New York, 1964.

[19] J. B. Keller. Surface tension force on a partly submerged body. Phys. Fluids, 10:3009-3010, 1998.

[20] P. Lancaster and K. Salkauskas. Surfaces generated by moving least squares methods. Mathematics of Computation, 37(155):pp. 141-158, 1981.

[21] J. J. Benito, F. Urena, L. Gavete, and R. Alvarez. An h-adaptive method in the generalized finite differences. Computer Methods in Applied Mechanics and Engineering, 192(5-6):735 - 759, 2003.

[22] J. C. Loudet, A. M. Alsayed, J. Zhang, and A. G Yodh. Capillary interactions between anisotropic colloidal particles. Phys. Rev. Lett., 94:018301, 2005. 



\section{RECENT REPORTS}

47/11 Effects of demographic noise on the synchronization of a metapopulation in a fluctuating environment

48/11 High order weak methods for stochastic differential equations based on modified equations

Abdulle

Cohen

Vilmart

Zygalakis

49/11 The kinetics of ice-lens growth in porous media

Style

Peppin

50/11 Wound healing angiogenesis: the clinical implications of a simple mathematical model

Flegg

Byrne

Flegg

McElwain

51/11 Wound healing angiogenesis: the clinical implications of a simple mathematical model

Du

Gunzburger

Lehoucq

Zhou

52/11 Image Inpainting based on coherence transport with Adapted dis-

März tance functions

53/11 Surface growth kinematics via local curve evolution

Moulton

Goriely

54/11 A multiple scales approach to evaporation induced Marangoni convection

Hennessey

Münch

55/11 The dynamics of bistable liquid crystal wells

Luo

Majumdar

Erban

56/11 Real-Time Fluid Effects on Surfaces using the Closest Point Method

Auer

Macdonald

Treib

Schneider

Westermann

$57 / 11$ Isolating intrinsic noise sources in a stochastic genetic switch

Newby

58/11 Riemann-Cartan Geometry of Nonlinear Dislocation Mechanics

Yavari

Goriely

59/11 Helices through 3 or 4 points?

Goriely

Neukirch

Hausrath

60/11 Bayesian data assimilation in shape registration

Cotter

Cotter

Vialard

61/11 Asymptotic solution of a model for bilayer organic diodes and solar cells

Richardson

Please

Kirkpatrick

$62 / 11$ Neural field model of binocular rivalry waves Bressloff 
63/11 Front propagation in stochastic neural fields Bressloff

Webber

64/11 Stability estimates for a twisted rod under terminal loads: a threedimensional study

Majumdar

Prior

Goriely

65/11 Adaptive Finite Element Method Assisted by Stochastic Simula-

Cotter tion of Chemical Systems

Vejchodsky

Erban

66/11 On the shape of force-free field lines in the solar corona Prior

Berger

$67 / 11$ Tear film thickness variations and the role of the tear meniscus

Please

Fulford

Fulford

Collins

68/11 Comment on "Frequency-dependent dispersion in porous media"

Davit

Quintard

69/11 Molecular Tilt on Monolayer-Protected Nanoparticles

Giomi

Bowick

Ma

Majumdar

Copies of these, and any other OCCAM reports can be obtained from:

Oxford Centre for Collaborative Applied Mathematics Mathematical Institute

24 - 29 St Giles'

Oxford

OX1 3LB

England

www.maths.ox.ac.uk/occam 